\title{
A NOVEL ENERgY EFFICIENT ROUTING ALGORITHM FOR WIRELESS SENSOR NETWORKS USING SINK MOBILITY
}

\author{
Deepa V. Jose ${ }^{1}$ and Dr.G.Sadashivappa ${ }^{2}$ \\ ${ }^{1}$ Department of Computer Science, Christ University, Bangalore, India \\ ${ }^{2}$ Department of Telecommunication Engg, R.V College of Engineering, Bangalore, India
}

\begin{abstract}
There are numerous applications for wireless sensor networks which are inevitable now a day in our daily life. Majority of such applications which use wireless sensor networks will be in areas where the direct human intervention is impossible. So the limited energy available in such sensors is a threat for prolonging the life of the entire network. The need of energy efficiency in wireless sensor networks is a hot research topic in which lot of new strategies for improvement in energy efficiency has been sought after. As communication process consumes more energy, an energy efficient routing strategy can probably reduce the energy consumption to a great extend. This paper gives an overview of the different routing techniques in which mobile sinks are used to facilitate the routing process which can effectively reduce the energy use. A new routing strategy with mobile sinks and a static sink is proposed and is compared based on the matrices life time and average energy of the nodes with the existing Shortest Hop path (SH) algorithm. The simulation results shows the proposed algorithm is more energy efficient than the existing one.
\end{abstract}

\section{KEYWORDS}

Energy Efficiency, Mobile Sinks, Routing Protocols, Sensor Nodes, Static Sinks, Wireless Sensor Networks.

\section{INTRODUCTION}

Wireless Sensor Networks (WSNs) usually consists of a large number of small sensor nodes interconnected that offers the unique ability to monitor the physical world accurately. WSNs have gained a lot of interest in the field of research nowadays due to its varied applications in diverse fields that require surveillance and monitoring that have become inevitable in our daily life. But the main drawback of such sensors are their resource constrained nature mainly in the power backup capabilities of sensor nodes and as a result a number of issues have emerged out of which energy efficiency is an important matter of concern.

WSN sensors are expected to be remotely deployed in unattended environments. The distributed nature, dynamic topology and resource constraints of WSNs introduces very special requirements in routing protocols that should be met as majority of the energy is usually utilized for routing. The issues faced by sensor networks are related to routing, network failure due to energy holes or energy drainage etc in which the problem of energy loss has to handled carefully as it can become the cause for so many other issues. So improving the energy efficiency of sensor nodes is very important. The most important feature of a routing protocol, in order to be efficient for WSNs, is reduced energy consumption and their by the extension of the network's lifetime. During the recent years, many energy efficient routing protocols have been proposed for WSNs. But in majority of them the mobility factor is not taken into consideration. In a WSN scenario there can 
International Journal of Wireless \& Mobile Networks (IJWMN) Vol. 6, No. 6, December 2014

be different types of mobility like the mobility of the nodes, sink mobility and the event or target mobility. Literature have shown that energy can be saved in a high level if sink mobility is achieved. In WSNs the criteria of designing routing etc depends on the application under consideration. The sink mobility concept can be introduced in application like smart home environment or medical applications like patient monitoring without many complications. But the number of sinks to be introduced, the positioning of the sinks, the mobility or pathway of the sink, it's velocity, coverage area etc are the different factors which has to be determined with care to have efficient results by attaining better energy utilization.

Wireless sensor Networks (WSNs) which consists of numerous tiny sensors, deployed randomly is exploited in copious applications now a day. The small size of these sensors helps to get it embedded in other devices very slickly which led to the use of sensors in variety of surveillance and monitoring applications, health and medical fields, engineering and automation and so on. The small size of the sensors is a boon as well as a hitch. Each sensor node will contain a sensing unit, a processing unit, memory and a power source. Because of its small size, deployment is easy but other processing, memory and power backup capacities have to be sacrificed. The block diagram of the components of a sensor node will be as represented in Figure1.

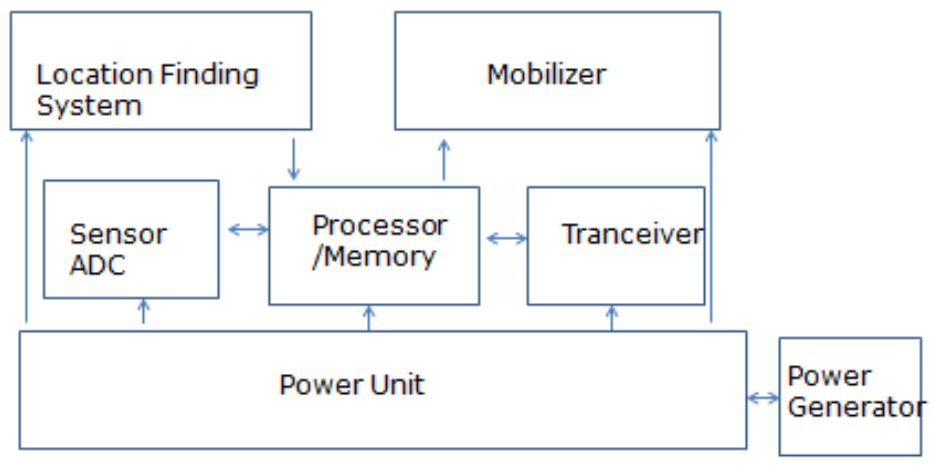

Figure 1. Components of a sensor node

The location finding system and the mobilizer are optional components and will be avoided usually as it can lead to the increase in size and cost of the entire system. The sensor ADC converts the sensed analog signal to their digital form. There will be a small processor and a memory for the processing and storage functions. The transceiver unit performs the transmission and reception of the signals. The power source will be usually batteries. The batteries in the wireless sensors are irreplaceable and of limited capacity.

Many energy harvesting techniques have been developed so as to frequently charge the sensors but it requires an additional hardware resource which leads to the increase in the cost and size of the sensor nodes which makes it difficult for many applications to adopt the use of WSNs. Above all the application of WSNs will be in areas where the human intervention is difficult or usually impossible. If anyone node dies off in the network, it can lead to the breakage of the entire network and the application can be futile. So the only possible solution is to efficiently use the available energy so as to extend the life time of the entire network.

The sensor nodes will be deployed randomly in an area where the sensing or monitoring has to happen, called the sensor field. Some nodes can act as sensor as well router which will collect data from all the nodes and passes on to the sink which is deployed outside the sensor filed. From there the data can be accessed by the users using the different available technologies. A general structure of the WSN is given in Figure 2. 


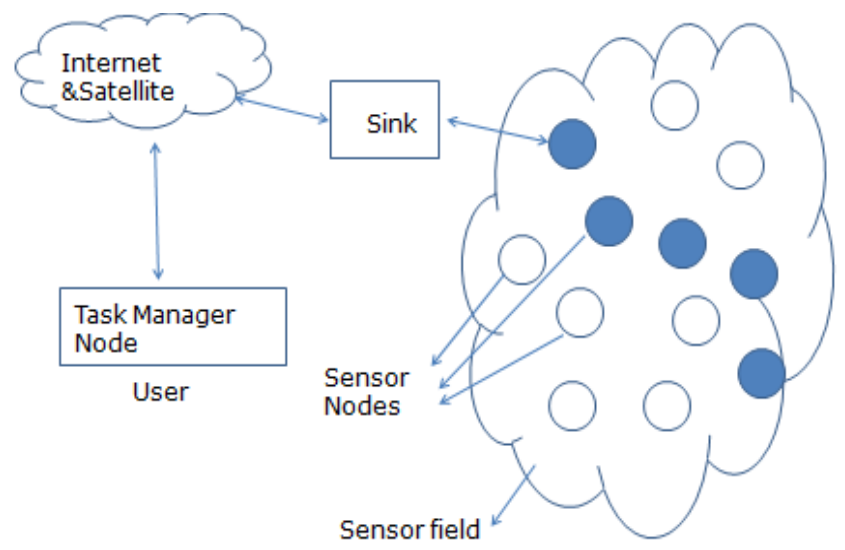

Figure 2. Wireless sensor network

Energy efficiency in WSNs is a hot research area where lot of methods to improve the energy efficiency is researched. There are different methods or techniques adopted to reduce the energy consumption or to effectively use the available energy to the maximum extent possible. Some of them are adopting different node deployment strategies, applying duty cycling concepts, including the mobility of some nodes which can act as data collector and router, changing the role of nodes accordingly etc. Each method will be appropriate for a specific application. According to the literature the majority of the energy is consumed for communication rather than that of processing and sensing. So the need of an energy efficient routing protocol is very important to extend the network life time. The energy efficient routing protocols falls under the hierarchical category where the clustering concept is used [1] [16] [17].

This paper proposes a new energy efficient routing algorithm which uses multiple mobile sinks and duty cycling concepts to improve the energy efficiency. The paper is organized into different sections. An overview of the related work in which the different papers related to mobile sinks and their outcome is discussed along with the duty cycling concepts is given in Section 2 followed by Section 3 describing the proposed algorithm .Section 4 shows the comparison with existing SH protocol with the simulation results based on the number of live nodes and the average residual energy of the nodes after the simulation period. Conclusion and future enhancements are mentioned in Section 5.

\section{REVIEW OF LITERATURE}

A mathematical model to find the best position of the sink in the network, which can reduce the energy for data transmission between the source and the sink is described in [2] .They have used the concept of cooperative routing scheme, shortest path or direct routing to achieve energy efficiency. In [3] the effect of sink mobility in prolonging the network life time is studied by suggesting a framework and a mathematical model to check the mobility of the sink with the delay constraints.

The authors in [4] say that when the sink moves along the area, the frequent location updates of it will lead to higher consumption of energy by sensors. They propose an efficient algorithm named IAR, an Intelligent Agent-based Routing protocol. They claim that their algorithm gives better performance and this scheme effectively supports mobility of the sink without much trouble. 
International Journal of Wireless \& Mobile Networks (IJWMN) Vol. 6, No. 6, December 2014

Lino et al. in [5] focus on mobility supported applications and proposes a new algorithm called MRLG- the Mobile-sink Routing that works better in large sensor networks in grid topology even though it supports other topologies also. Assumptions made are the number of nodes will not increase randomly, the sensor nodes will be static and the sink is freely movable. The authors declare that results are compared with Collection Tree Protocol (CTP) and it outperforms CTP in terms of delay, packet delivery ratio, end- and other routing overheads.

The authors in [6] consider the overload of frequent location updates due to sink mobility and introduce a novel data gathering mechanism with mobile sinks. Simulation results show that this method reduces the energy consumption and data loss considerably. Data aggregation mechanism using mobile sink nodes in proposed in [7]. Here nodes are allowed to form as clusters using Gravitational Search Algorithm and no Cluster Head election takes place but the role of Cluster Heads is taken over by the introduced mobile sink nodes to perform data gathering, data aggregation as well as communication operations which results in increasing the lifetime of the sensor nodes. Simulations are carried out using Network Simulator with parameters energy consumption and network lifetime.

In this paper [8] a new hierarchical routing protocol Layer Based Low Energy Adaptive Clustering Hierarchy (LB-LEACH) is proposed to increase the lifetime of WSN in which layer factor is considered as a cluster head selection parameter to improve the performance of cluster head selection algorithm proposed in LEACH, EECHS and energy aware LEACH. For data collection, a static sink and multiple mobile sinks is introduced. Authors claim LB-LEACH performs better than LEACH, Energy aware LEACH and EECHS.

Yu Gu et al in their work [9] suggests a framework for analyzing the mobility of the sink. They claim that simulations show the advantages of using a mobile sink and its influence of the factors like delay and number of nodes. In [10] the authors aim to reduce the energy consumption in multihop data retrieval and balance the energy among the entire sensor nodes which leads to lifetime elongation. Here the cluster heads filter out the redundant data and passes to nodes with residual energy close to mobile sinks. Simulation results show this method is far better than the existing methods.

Duty cycling is an important way to reduce energy consumption. In order to efficiently use the limited available energy of the sensor nodes a thorough study of duty-cycling concept combined with data aggregation techniques is done in this paper [11]. The authors uses a specific method using Shortest path tree for data aggregation which will be having uniform load and good latency along with duty cycling. Simulations are done which proves the efficiency of their work.

In [12] the authors say that the immediate area surrounding the sink is the major site which limits the network lifetime due to the heavy traffic-flow in that area. So their attempt is to improve the energy efficiency of area around the sink which the authors claims with the duty cycling introduced will improve life time to a better extend. In order to prove the efficacy of the approach adopted, a detailed theoretical analysis and simulation results have also been provided.

The authors in [13] have studied the problem of minimizing the total transmission power for reliable data dissemination in duty cycled WSNs in multicast or broadcast. They propose the novel concept of Time-Reliability-Power (TRP) space as a general data structure for designing data dissemination algorithms in WSNs. According to them through simulations are done which evidently demonstrate the efficiency of their proposed algorithms. According to Jie Hao et al in [14] sleep scheduling is a very common method to reduce energy consumption. In this article the authors addresses the basic routing protocol design issues for duty cycled networks, classification 
International Journal of Wireless \& Mobile Networks (IJWMN) Vol. 6, No. 6, December 2014

of existing protocols based on different design criteria in this area, their working and the pros and cons along with some future directions.

Lutz, K and Konig in [15] say about the hardware concepts related to energy reduction. This paper also investigates about the different circuit design criteria including the duty cycling. This is more or less an application specific idea but can be adopted to reduce energy usage. In [16] the authors have addressed the problems of the cluster head related to uneven load and the consequence related to this .In [17] a new routing strategy where multiple mobile sinks and targets are involved is proposed with a comparison with the existing scheme.. A scheme to conserve energy with sink mobility wherein the sink moves based on the energy of the cluster head is proposed in [18].

The authors in [19] suggest methods to remove the overheads and additional wastage of energy due to mobility of the sink and propose a solution for the triangular routing problem. They claims that the proposed method proves to be efficient both by analytical and simulation techniques. In [20] a mobile data collector based protocol is proposed and a comparison is done to prove its efficacy. Using the ant colony algorithms an efficient routing strategy and the benefits of mobile sinks is done in [21].

\section{DESCRIPTION OF THE PROPOSED ALGORITHM}

Among the different categories of existing routing protocols the hierarchical model is the one which concentrates on energy efficiency. The protocols in this criterion use the clustering concept. In clustering there will be different levels of clusters so that all the nodes need not take part in the entire data transmission all the time. There will be a cluster head which will accept all the nodes data under its leadership, aggregates it and send to the next higher level.

The proposed algorithm uses the clustering concept in which the sinks are made mobile. Mobility of the sink node can be made randomly or based on a predetermined fashion. Clusters are formed on a special strategy wherein the residual energy of the nodes is taken into consideration.

The maximum utilization of the energy is achieved by incorporating duty cycles among the nodes. The nodes which are within the half range of a cluster head will be sharing the time slot for transmission. During that particular time slot only the specific node will be active and rest of the nodes will be in sleep mode thereby saving the energy. The data that is sensed by all the nodes will be sent to the mobile sink which reduces the number of hops for transmission to the destination sink which is made static. This also reduces the energy for transmission.

The sensor nodes are distributed randomly in the network. One sink is at fixed location. There are multiple mobile sinks which are under the control of the static sink. The path of movement of mobile sink is decided by the static sink. All the nodes in the network know the path to static sink. Mobile sinks have bigger communication range and can communicate with static sink directly. Initially the nodes send data to static sink.

The static sink receives data from the nodes and analyzes the behavior of nodes. It groups the nodes based on the behavior and creates the cluster. The duty cycle is distributed to the nodes in the cluster by the static sink and sent to the node. Based on the distribution of clusters, the static sink does the path planning for the mobile sinks and instructs the mobile sink to travel the path. Mobile sink travels in the path, stops at certain locations and collect data. 
International Journal of Wireless \& Mobile Networks (IJWMN) Vol. 6, No. 6, December 2014

To create cluster, node with similar behavior and within certain coverage area is grouped into one cluster. The behaviour of node is modelled using ARIMA and the model is compared to measure the similarity of node behaviour. The mobile sink stops at certain locations in the path and sends a broadcast packet to neighbourhood. Nodes receiving this broadcast packet will construct the route. The data it has will be sending to nearest mobile sink. Mobile sink will do the aggregation and send the aggregated packet to static sink.

The proposed algorithm is compared with the shortest hop path algorithm. The SH algorithm seems to be energy efficient since it takes the shortest hop to reach the destination. Here only the shortest hop is taken into consideration. But the energy level of the nodes is also a major factor to be addressed. The main drawback of this is the continuous use of the nodes in the shortest hop path to the destination which leads to the fast energy drainage of those nodes which will ultimately lead to the network failure. This can occur even when there are nodes with sufficient energy for data transmission which are never used at all. Since we are using the mobile sinks this problem is avoided and the number of hops is also reduced as the sink is mobile there by reducing the energy to a great extends.

\section{SIMULATION RESULTS}

The proposed algorithm is compared with the $\mathrm{SH}$ algorithm. The simulations are carried out using MATLAB. The simulation parameters are given in Table 1. The simulations are done based on the assumption that the mobile sinks are rechargeable and do not have any failure during the entire session. They will be able to move and reach each cluster head to collect the information.

Table 1.Simulation parameters

\begin{tabular}{|l|l|}
\hline No of Nodes & 100 \\
\hline Area of simulation & $100 * 100 \mathrm{~m}$ \\
\hline Range of Sensor & $10 \mathrm{~m}$ \\
\hline Initial Energy at sensor & 50 Joules \\
\hline Transmission Energy & .5 Joules \\
\hline Receiving Energy & .2 Joules \\
\hline No of mobile sink & 4 \\
\hline No of static sink & 1 \\
\hline Deployment Model & Random \\
\hline
\end{tabular}

The initial node deployment in the designated area according to the parameters given in Table. 1 is represented in Figure 3. Simulation is done by changing the parameters to prove the efficacy of the proposed algorithm. Simulations exhibited that the Mobile Sink Assisted routing, the 
International Journal of Wireless \& Mobile Networks (IJWMN) Vol. 6, No. 6, December 2014

proposed one, is better than the SH routing to a great extend in terms of number of live nodes, the average energy of the nodes and the packet delivery ratio within the simulation time allocated.

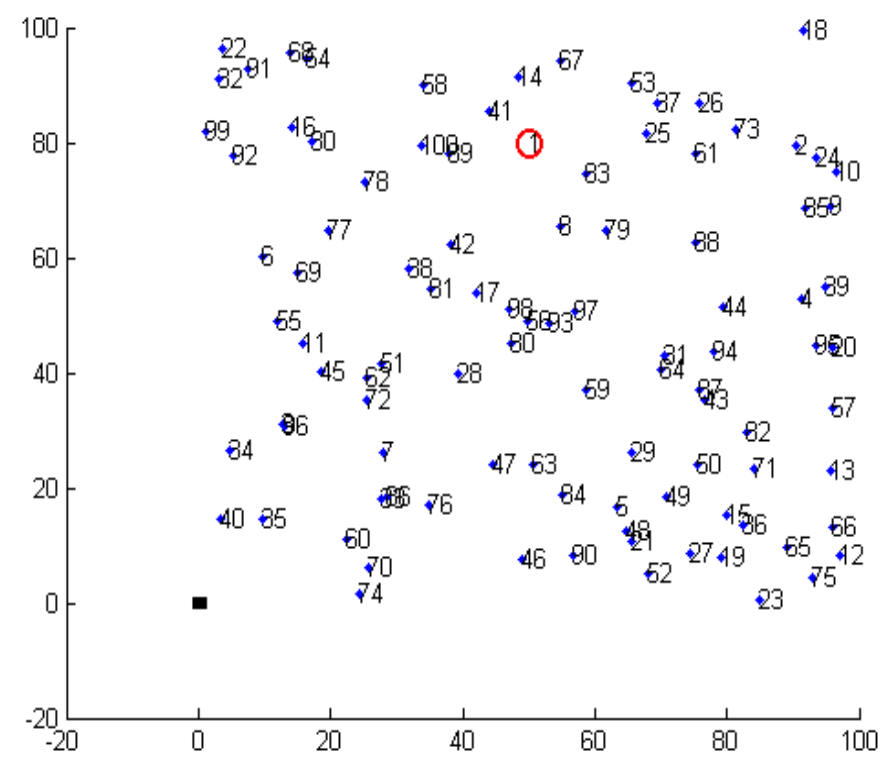

Figure 3. Initial node deployment

The cluster head selection scenario is represented in Figure 4.The formation of the cluster is done following the LEACH protocol. Based on the probabilistic function followed in LEACH the cluster heads are selected. They in turn broadcast this message to their neighbours. Based on the received signal strength each node forms a part of the cluster. After this stage the sinks are made mobile to different directions where they will collect the data from the respective cluster heads when they reach near each cluster head. The mobile sinks aggregates all the information and passes on to the static sink from where the user can access the information.

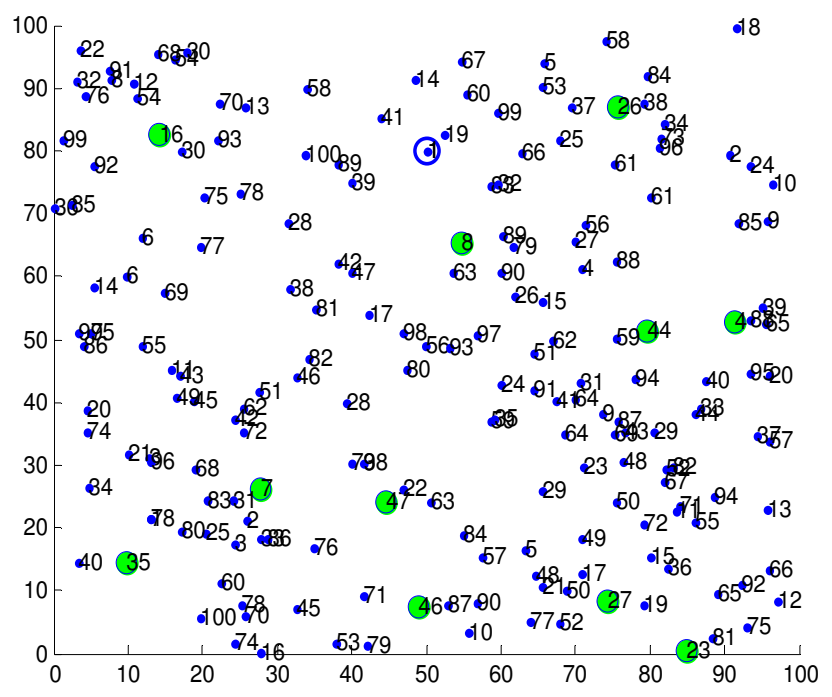

Figure 4. Clustered sensor network 
The simulation results based on the parameters number of live nodes, the average energy of each node and the packet delivery ratio for the proposed mobile sink assisted algorithm and $\mathrm{SH}$ algorithm is given below in Figure 5, Figure 6 and Figure 7 respectively.

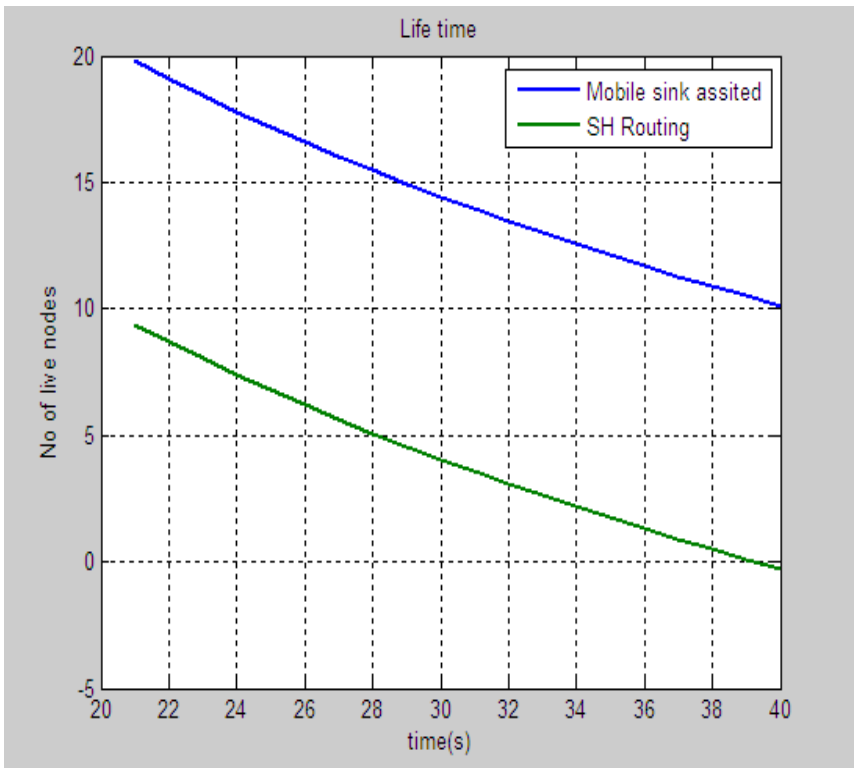

Figure 5. Life time

From the above figure it is evident that the number of live nodes drops to zero before the expiry of the simulation time in the case of SH. But in the case of mobile sink assisted almost double the nodes are alive during the simulation period which shows that the proposed algorithm have shown more than $50 \%$ improvement based on the number of live nodes i.e. the life time of the network. Figure 6 represents the average energy of the sensor nodes.

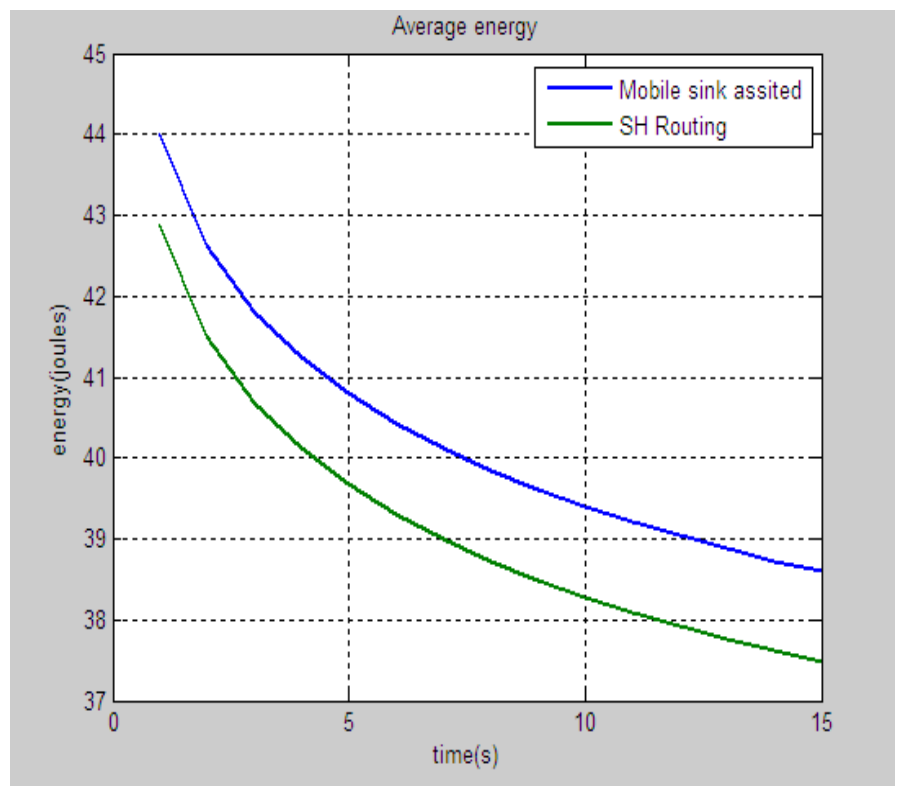


Figure 6. Average energy

It is evident that the proposed algorithm gives more than $10 \%$ improvement in the average energy level of the nodes compared to SH which in turn will lead to a better lifetime. Same way in the case of packet delivery ratio also a huge difference in the two approaches is clearly evident from the simulation result.

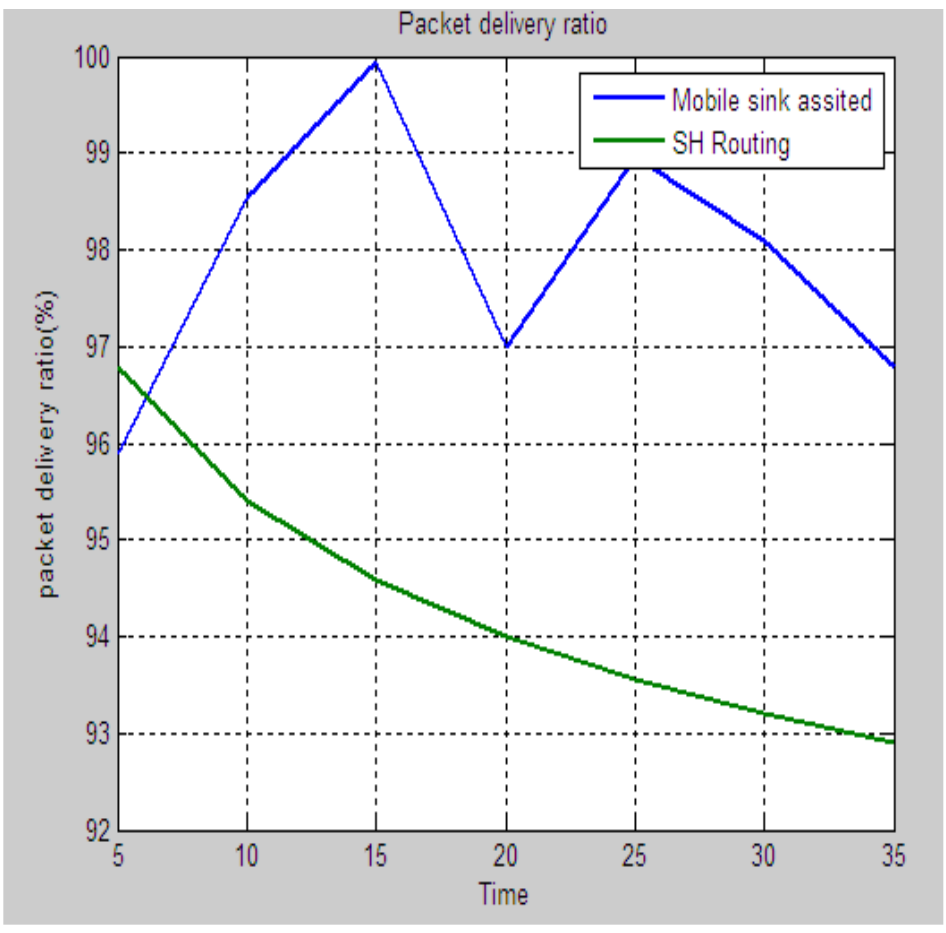

Figure 7. Packet delivery ratio

\section{CONCLUSION}

We are aware that energy efficiency is always a critical concern in wireless sensor networks even though lot of work related to this exists. Mobility of the nodes is a major issue in this field as the mobility can be of that of the sensor nodes or of the sinks. The proposed mobile sink assisted algorithm makes the sinks mobile so as to reduce the number of hops to the destination thereby reducing the energy consumption. The duty cycling introduced makes only specific nodes alive and all others to sleep mode which in turn will reduce the energy consumption. The proposed algorithm proves to have better life time compared to $\mathrm{SH}$ which is evident from the simulation results. The average energy of the nodes also is higher compared to SH. So it can be concluded that the proposed algorithm gives better life time and hence efficient than SH.

The future enhancement of this algorithm which is underway is to change the mobility structure of the mobile sinks along with the cluster formation strategy and comparing the results for other matrices like delay and throughput .Further the proposed mobile sink assisted algorithm will be compared with the existing energy efficient routing protocols to sturdily emphasize the efficiency of the proposed one.

\section{ACKNOWLEDGMENTS}


I sincerely thank Dr. G Sadashivappa for the inspiration rendered throughout. He is always dynamic and helpful enough to share his valuable expertise, knowledge and experience to make this research work a fruitful one. I thank my family and friends for their constant support and motivation.

\section{REFERENCES}

[1] Pantazis, Nikolaos A., Stefanos A. Nikolidakis, and Dimitrios D. Vergados. "Energy-efficient routing protocols in wireless sensor networks: A survey." Communications Surveys \& Tutorials, IEEE 15, no. 2 (2013): 551-591

[2] Sivsankari, H., R. Leelavathi, K. Shaila, K. R. Venugopal, S. S. Iyengar, and L. M. Patnaik. "Energy Efficient Adaptive Cooperative Routing (EEACR) with multiple sinks in Wireless Sensor Networks." In Industrial Electronics and Applications (ICIEA), 2012 7th IEEE Conference on, pp. 676-681. IEEE, 2012.

[3] Gu, Yu, Yusheng Ji, Jie Li, Biao Han, and Baohua Zhao. "Delay-bounded sink mobility in wireless sensor networks." In Communications (ICC), 2012 IEEE International Conference on, pp. 740-744. IEEE, 2012.

[4] JKim, Jae-Wan, Jeong-Sik In, Kyeong Hur, Jin-Woo Kim, and Doo-Seop Eom. "An intelligent agentbased routing structure for mobile sinks in WSNs." Consumer Electronics, IEEE Transactions on 56, no. 4 (2010): 2310-2316.

[5] Lino, C., Carlos T. Calafate, Arnoldo Diaz-Ramirez, Pietro Manzoni, and J-C. Cano. "An efficient solution offering sink mobility support in wireless sensor networks." In Wireless Telecommunications Symposium (WTS), 2012, pp. 1-9. IEEE, 2012.

[6] Faheem, Yasir, Saadi Boudjit, and Ken Chen. "Dynamic sink location update scope control mechanism for mobile sink Wireless Sensor Networks." In Wireless On-Demand Network Systems and Services (WONS), 2011 Eighth International Conference on, pp. 171-178. IEEE, 2011.

[7] Rejina Parvin, J., and C. Vasanthanayaki. "Gravitational Search Algorithm Based Mobile Aggregator Sink Nodes for Energy Efficient Wireless Sensor Networks." In Circuits, Power and Computing Technologies (ICCPCT), 2013 International Conference on, pp. 1052-1058. IEEE, 2013.

[8] Sen, Aditi, M. Das Gupta, and Debashis De. "Energy efficient layered cluster based hierarchical routing protocol with Dual Sink." In Computers and Devices for Communication (CODEC), 2012 5th International Conference on, pp. 1-4. IEEE, 2012.

[9] Gu, Yu, Yusheng Ji, Jie Li, and Baohua Zhao. "ESWC: efficient scheduling for the mobile sink in wireless sensor networks with delay constraint." Parallel and Distributed Systems, IEEE Transactions on 24, no. 7 (2013): 1310-1320.

[10] Konstantopoulos, Charalampos, Grammati Pantziou, Damianos Gavalas, Aristides Mpitziopoulos, and Basilis Mamalis. "A rendezvous-based approach enabling energy-efficient sensory data collection with mobile sinks." Parallel and Distributed Systems, IEEE Transactions on 23, no. 5 (2012): 809817.

[11] ZChen, Lei, Geng Yang, Zhengyu Chen, Haiyong Wang, and Jian Xu. "A load-balanced data aggregation scheduling for duty-cycled wireless sensor networks." In Proceedings of the 2012 IEEE 4th International Conference on Cloud Computing Technology and Science (CloudCom), pp. 888893. IEEE Computer Society, 2012.

[12] Rout, Rashmi Ranjan, and Soumya K. Ghosh. "Enhancement of lifetime using duty cycle and network coding in wireless sensor networks." Wireless Communications, IEEE Transactions on 12, no. 2 (2013): 656-667.

[13] Han, Kai, Jun Luo, Liu Xiang, Mingjun Xiao, and Liusheng Huang. "Achieving Energy Efficiency and Reliability for Data Dissemination in Duty-Cycled WSNs." 1-1.

[14] Hao, Jie, Baoxian Zhang, and Hussein T. Mouftah. "Routing protocols for duty cycled wireless sensor networks: A survey." Communications Magazine, IEEE 50, no. 12 (2012): 116-123.

[15] Lutz, Kai, and Andreas Konig. "Minimizing power consumption in wireless sensor networks by dutycycled reconfigurable sensor electronics." In Intelligent Solutions in Embedded Systems (WISES), 2010 8th Workshop on, pp. 97-102. IEEE, 2010. 
International Journal of Wireless \& Mobile Networks (IJWMN) Vol. 6, No. 6, December 2014

[16] Dali Wei; Yichao Jin; Vural, S.; Moessner, K.; Tafazolli, R.; , "An Energy-Efficient Clustering Solution for Wireless Sensor Networks," Wireless Communications, IEEE Transactions on , vol.10, no.11, pp.3973-3983, November 2011

[17] Yuan-Po Chi; Hsung-Pin Chang;, "TARS: An Energy-Efficient Routing Scheme for Wireless Sensor Networks with Mobile Sinks and Targets," Advanced Information Networking and Applications (AINA), 2012 IEEE 26th International Conference on , vol., no., pp.128-135, 26-29 March 2012.

[18] Yuan Xun-Xin; Zhang Rui-Hua, "An Energy-Efficient Mobile Sink Routing Algorithm for Wireless Sensor Networks," Wireless Communications, Networking and Mobile Computing (WiCOM), 2011 7th International Conference on, vol., no., pp.1,4, 23-25 Sept. 2011.

[19] Jae-Wan Kim; Doo-Seop Eom, "An agent-based routing algorithm with low overhead for mobile sinks in wireless sensor networks," Advanced Communication Technology, 2009. ICACT 2009. 11th International Conference on, vol.02, no., pp.1156,1159, 15-18 Feb. 2009.

[20] Arshad, M.; Aalsalem, M.Y.; Siddqui, F.A., "Energy efficient cluster based routing scheme for mobile Wireless Sensor Networks," Intelligent and Advanced Systems (ICIAS), 2014 5th International Conference on , vol., no., pp.1,6, 3-5 June 2014.

[21] Wang Jia; He Zhimin, "Ant Colony Optimization Algorithms-Based Routing Planning of Mobile Sink," KAM, 2011 Fourth International Symposium on , vol., no., pp.28,31, 8-9 Oct. 2011.

\section{Authors}

Ms.Deepa V. Jose is currently pursuing Ph.D. in Computer Science from Christ University Bangalore. Her research interests are in the field of Wireless sensor networks and Cryptography.

Dr.G Sadashivappa have more than twenty five years of experience in teaching research and industry. His research interests include Fibre optic communication, Signal Processing and Applications.
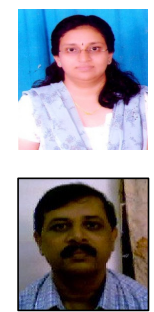